\title{
MASS-LOSS RATES OF OH/IR STARS
}

\author{
Kyung-Won Suh And Young-Joo Kwon \\ Department of Astronomy and Space Science, Chungbuk National University, Cheongju 361-763, Korea \\ E-mail : kwsuh@chungbuk.ac.kr \\ (Received October 17, 2013; Accepted November 5, 2013)
}

\begin{abstract}
We compare mass-loss rates of $\mathrm{OH} / \mathrm{IR}$ stars obtained from radio observations with those derived from the dust radiative transfer models and IR observations. We collect radio observational data of $\mathrm{OH}$ maser and CO line emission sources for a sample of $1533 \mathrm{OH} / \mathrm{IR}$ stars listed in Suh \& Kwon (2011). For $1259 \mathrm{OH}$ maser, $76 \mathrm{CO}(\mathrm{J}=1-0)$, and $55 \mathrm{CO}(\mathrm{J}=2-1)$ emission sources, we compile data of the expansion velocity and mass-loss rate. We use a dust radiative transfer model for the dust shell to calculate the mass-loss rate as well as the IR color indices. The observed mass-loss rates are in the range predicted by the theoretical dust shell models corresponding to $\dot{M}=10^{-8} M_{\odot} / y r-10^{-4} M_{\odot} / y r$. We find that the dust model using a simple mixture of amorphous silicate and amorphous $\mathrm{Al}_{2} \mathrm{O}_{3}$ (20\% by mass) grains can explain the observations fairly well. The results indicate that the dust radiative transfer models for IR observations generally agree with the radio observations. For high mass-loss rate OH/IR stars, the mass-loss rates obtained from radio observations are underestimated compared to the mass-loss rates derived from the dust shell models. This could be because photon momentum transfer to the gas shell is not possible for the physical condition of high mass-loss rates. Alternative explanations could be the effects of different dust-to-gas ratios and/or a superwind.
\end{abstract}

Key words : stars: AGB stars — infrared: stars — circumstellar matter — masers — dust: extinction

\section{INTRODUCTION}

The $\mathrm{OH} / \mathrm{IR}$ stars are generally believed to be the last evolutionary phase of an oxygen-rich asymptotic giant branch (AGB) star emitting $\mathrm{OH}$ maser at 1612 $\mathrm{MHz}$. OH/IR stars are characterized by the Mira-type long-period variable stars (LPVs) with large amplitude pulsation. Shock waves produced by the strong pulsation and radiation pressure on newly formed dust grains drive dusty stellar winds with high mass-loss rates $\left(10^{-8}-10^{-4} M_{\odot} / y r\right)$. $\mathrm{OH}, \mathrm{SiO}$, and $\mathrm{H}_{2} \mathrm{O}$ masers have been found to be associated with O-rich AGB stars (e.g., Kwon \& Suh 2012). OH/IR stars typically show $10 \mu \mathrm{m}$ and $18 \mu \mathrm{m}$ features in emission or absorption which suggest the presence of silicate dust grains in the outer envelopes around them. Further investigations showed that there are more dust species (e.g., $\mathrm{Al}_{2} \mathrm{O}_{3}$ and water-ice).

The AGB phase is characterized by the relatively rapid onset of greatly enhanced mass-loss, commonly referred to as the superwind phase (Renzini 1981). As AGB stars evolve, thermal pulses due to internal helium shell flashes have been hypothesized as a major cause of these episodes of greatly enhanced mass-loss (Wood 1990; Suh \& Jones 1997). The mass-loss rate from an AGB star must increase by at least a factor of 10 for the duration of the superwind. We expect one thermal pulse about every $10^{4}-10^{5}$ years that endures

Corresponding Author: K.-W. Suh for a few hundred years (Iben 1981; Vassiliadis \& Wood 1993).

In this paper, we investigate the relations among the parameters to determine the mass-loss rates for a sample of $1533 \mathrm{OH} / \mathrm{IR}$ stars listed in Suh \& Kwon (2011). For the $\mathrm{OH} / \mathrm{IR}$ stars, we compare the mass-loss rates and expansion velocities obtained from radio observations with the dust mass-loss rates and IR colors derived from dust radiative transfer models and IR observations.

\section{SAMPLE STARS}

Radio observations of an $\mathrm{OH} / \mathrm{IR}$ star may provide useful information to obtain the expansion velocity of the outer shell and the mass-loss rate. $\mathrm{OH}$ molecules produce a characteristic double-peak emission line from the shell of gas expanding away from the star. The difference in the velocities of the two peaks gives the expanding velocity of the gas. We need to know the brightness of the maser emission and the distance of the object as well as the expansion velocity to obtain the mass-loss rate (e.g., Zhang et al. 2010).

We collect $V_{\text {exp }}$ data for $1912 \mathrm{OH}$ maser sources from 11 papers and 1259 maser sources can be identified to be OH/IR stars using the list of $1533 \mathrm{OH} / \mathrm{IR}$ stars in Suh \& Kwon (2011). In Table 1, we list the number of data for each reference. The mass-loss rate $(\dot{M})$ data are available for $100 \mathrm{OH} / \mathrm{IR}$ stars. 
$\mathrm{CO}$ emission lines are available to estimate the massloss rate as well as the expanding velocity. We list the CO emission sources and the number of IRAS counterparts in Table 2. Some CO sources show a composite CO profile: a narrow component superposed on a broader one at the same stellar velocity. Knapp et al. (1998) interpret this profile as a two successive winds having different mass-loss rates and outflow velocities. In this paper, we take the broad component only. For $76 \mathrm{CO}(\mathrm{J}=1-0)$ and $55 \mathrm{CO}(\mathrm{J}=2-1)$ emission sources, we compile data of the expansion velocity and massloss rate. For some objects which were observed more than one time to obtain $V_{\text {exp }}$, we use an average value of $V_{\text {exp. }}$.

\subsection{Comparison of Expansion Velocities}

There are $53 \mathrm{OH} / \mathrm{IR}$ stars which have both $\mathrm{OH}$ and $\mathrm{CO}(\mathrm{J}=1-0$ or $\mathrm{J}=2-1)$ sources and $47 \mathrm{OH} / \mathrm{IR}$ stars which have both $\mathrm{CO}(\mathrm{J}=1-0)$ and $\mathrm{CO}(\mathrm{J}=2-1)$ sources. In Fig. 1 , we plot the radial velocities of central star $\left(\mathrm{V}_{L S R}\right)$ and expanding velocities $\left(\mathrm{V}_{\exp }\right)$ of envelope obtained from $\mathrm{OH}$ maser and $\mathrm{CO}$ emission lines.

For $V_{L S R}$ (the upper panel of Fig. 1), $V_{L S R}$ from $\mathrm{CO}(\mathrm{J}=1-0)$ emission sources show a strong correlation (the correlation coefficient $\mathrm{R}$ is near from 1 ) with the ones from $\mathrm{CO}(\mathrm{J}=2-1)$ observations $\left(V_{L S R, C O(2-1)}\right.$ $\left.=V_{L S R, C O(1-0)}-0.03, \mathrm{R}=0.999\right) . \quad$ Also, $V_{L S R}$ from $\mathrm{CO}(\mathrm{J}=1-0$ or $\mathrm{J}=2-1)$ emission sources show a strong correlation with those from $\mathrm{OH}$ maser observations $\left(V_{L S R, O H}=V_{L S R, C O}-1.22, \mathrm{R}=0.997\right)$. The strong correlations of $V_{L S R}$ mean that the stellar and circumstellar structure maintain spherical symmetry despite variations of the physical structure in time.

On the lower panel of Fig. 1, $V_{\text {exp }}$ obtained from two $\mathrm{CO}$ lines $(\mathrm{J}=2-1$ and $\mathrm{J}=1-0)$ show comparatively a mild correlation $\left(V_{\exp , C O(2-1)}=V_{\exp , C O(1-0)}+0.04\right.$, $\mathrm{R}=0.953$ ). Olofsson et al. (1993) found that there is no significant difference between the two estimates of the gas expanding velocity from $\mathrm{CO}$ lines of $\mathrm{J}=2-1$ and

Table 1.

$\mathrm{OH}$ maser sources

\begin{tabular}{lcc}
\hline \hline References & $V_{\text {exp,OH }}$ & $V_{\text {exp }, O H}$ and $\dot{M}$ \\
\hline te Lintel Hekkert et al. (1991) & 641 & \\
Eder et al. (1988) & 169 & \\
Lewis et al. (1990) & 71 & \\
Chengalur et al. (1993) & 115 & \\
Lewis (1994) & 39 & \\
Sivagnanam et al. (1990) & 27 & \\
Le Squeren et al. (1992) & 109 & \\
David et al. (1993) & 126 & \\
Sevenster et al. (1997a) & 248 & \\
Sevenster et al. (1997b) & 166 & \\
Sevenster et al. (2001) & 276 & 110 \\
Zhang et al. (2010) & 0 & 100 \\
\hline IRAS sources & $1912(1421)$ & \\
OH/IR stars & $1745(1259)$ & \\
\hline
\end{tabular}

The numbers in the parenthesis refer to the numbers of stars.
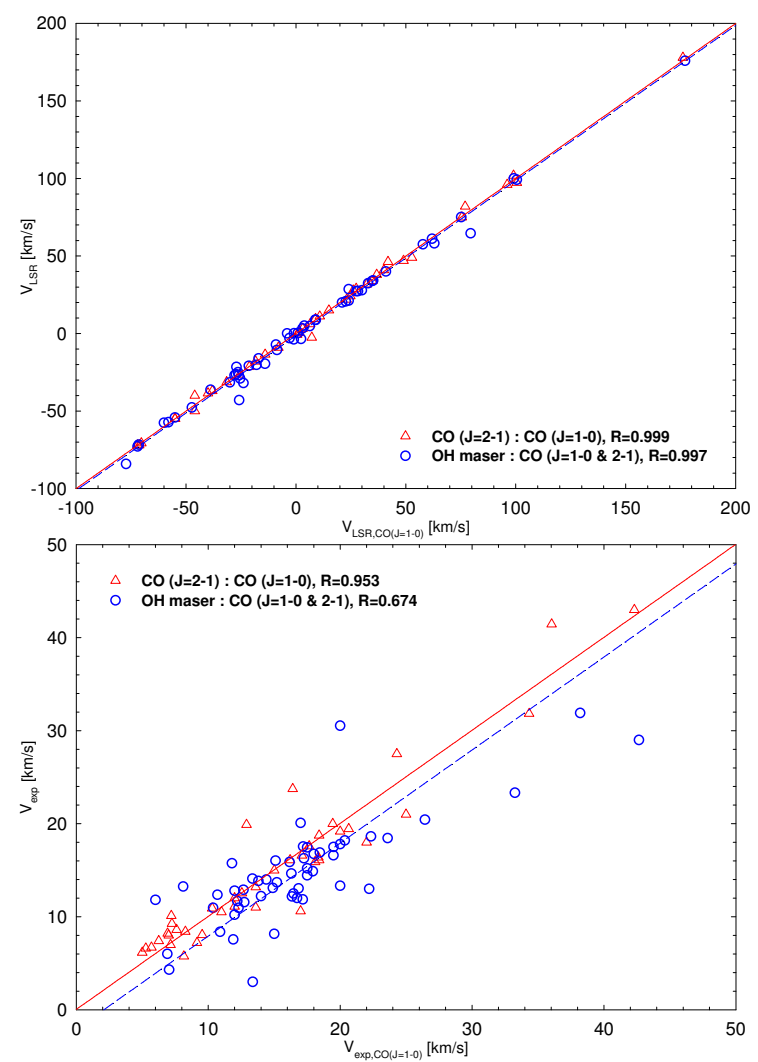

Fig. 1. - Comparison of expansion velocities between $\mathrm{OH}$ masers and CO lines.

$\mathrm{J}=1-0$.

The expanding velocities derived from $\mathrm{OH}$ maser and $\mathrm{CO}$ lines show a weak correlation $\left(V_{\text {exp }, O H}=\right.$ $\left.V_{\text {exp }, C O}-2.08, \mathrm{R}=0.674\right)$ displaying considerable scatter. This could be due to different locations of the sources or different pulsation phases.

\subsection{The $I R A S$ and $2 M A S S$ Data}

IR colors ([12]-[25] and $K$-[12]) are useful to characterize the dust shells around $\mathrm{OH} / \mathrm{IR}$ stars. By using version 2.1 of the Infrared Astronomical Satellite $(I R A S)$ Point Source Catalog (PSC), we obtain useful photometric data at two bands (12 and $25 \mu \mathrm{m}$ ) for $1533 \mathrm{OH} / \mathrm{IR}$ stars.

We use the two micron all sky survey $(2 M A S S)$ data at $K(2.17 \mu \mathrm{m})$ band. We cross-identify the $2 M A S S$ source by finding the nearest in position within 10 arcsec. For a number of sample stars which do not have good quality $2 M A S S$ data at $K$ band, we use other available $K$ band data listed in Suh \& Kwon (2011).

The color index is defined as

$$
M_{\lambda 1}-M_{\lambda 2}=2.5 \log _{10} \frac{F_{\lambda 2} / Z M C_{\lambda 2}}{F_{\lambda 1} / Z M C_{\lambda 1}}
$$

where $Z M C_{\lambda i}$ means the zero magnitude calibration 
Table 2.

$\mathrm{CO}$ emission line sources

\begin{tabular}{lccc}
\hline \hline References & $V_{\text {exp }, C O(J=1-0)}$ & $V_{\text {exp }, C O(J=2-1)}$ & $V_{\text {exp }, C O}$ and $\dot{M}$ \\
\hline Loup et al. (1993) & 524 & 292 & 36 \\
Nyman et al. (1992) & 163 & 0 & \\
Groenewegen \& de Jong (1998) & 0 & 4 & \\
Knapp et al. (1998) & 0 & 28 & 20 \\
Groenewegen et al. (1999) & 24 & 40 & \\
Kemper et al. (2003) & 0 & 14 & 18 \\
Winters et al. (2003) & 49 & 60 & $74(74)$ \\
De Beck et al. (2010) & 56 & 0 & $74(74)$ \\
\hline IRAS sources & $816(373)$ & $438(308)$ & $87(55)$ \\
OH/IR stars & $183(76)$ & $87(76)$ & \\
\hline
\end{tabular}

The numbers in the parenthesis refer to the numbers of stars.

at given wavelength $(\lambda i)$ (see Suh \& Kwon 2011 for details).

We use only those objects with good quality data at any wavelength.

\section{THEORETICAL DUST SHELL MODELS}

We perform theoretical radiative transfer model calculations using DUSTY code developed by Ivezić \& Elitzur (1997) for a spherically symmetric dust shell.

We assume that the dust density distribution is continuous $\left(\rho \propto r^{-2}\right)$ from the dust condensation radius $\left(R_{i n}\right)$ to the outer radius $\left(R_{\text {out }}\right)$ and the mass-loss rate $(\dot{M})$ and the envelope expansion velocity $\left(V_{\text {exp }}\right)$ are constant. Then the mass-loss rate is given by

$$
\dot{M}=4 \pi r^{2} \rho V_{\text {exp }} \simeq 4 \pi R_{\text {in }}^{2} \rho_{o} V_{\text {exp }},
$$

where $\rho_{o}$ is the material density at $R_{i n}$. The dust shell optical depth $\left(\tau_{\lambda}\right)$ is given by

$$
\tau_{\lambda}=\int \kappa_{\lambda} \rho_{d} d r \simeq \kappa_{\lambda} \rho_{d} R_{i n}
$$

where $\kappa_{\lambda}$ is the dust extinction opacity in $\mathrm{g}^{-1} \mathrm{~cm}^{2}$ and $\rho_{d}$ is the dust density at $R_{i n}$ which is given by

$$
\rho_{d}=\rho_{o} \delta
$$

where $\delta$ is the dust-to-gas ratio.

The outer radius of the dust shell is always taken to be $1 \times 10^{4}$ times the inner radius $\left(R_{i n}\right)$. The inner shell dust temperature $\left(T_{c}\right.$; the dust condensation temperature) is the dust temperature at $R_{i n}$. We assume that $T_{c}=1000 \mathrm{~K}$. Although AGB stars are pulsating, the overall continuous density distribution is believed to be maintained for a time scale larger than the pulsation period (e.g., Suh 1999; Suh 2004).

\subsection{Dust Shell Model Parameters}

We choose $10 \mu \mathrm{m}$ as the fiducial wavelength that sets the scale of the dust optical depth $\left(\tau_{10}\right)$ and compute models for various optical depths $\left(\tau_{10}=0.001-60\right)$.
For the central star, we assume that the luminosity is $1 \times 10^{4} L_{\odot}$ and the stellar blackbody temperature $\left(\mathrm{T}_{\text {star }}\right)$ is $2500 \mathrm{~K}$ for $\tau_{10} \leq 3,2000 \mathrm{~K}$ for $\tau_{10}>3$.

The dust-to-gas ratio $(\delta)$ is generally estimated to be 0.005 to 0.02 . Draine et al. (2007) derived the average dust-to-gas ratio in Milky way to be 0.01 and argued that the value tends to increase for a higher metallicity. The dust-to-gas ratio $(\delta)$ is assumed to be 0.01 .

For modeling dust shells around O-rich AGB stars, Suh \& Kwon (2011) found that the dust opacity using $\mathrm{Al}_{2} \mathrm{O}_{3}$ as well as silicate can improve the fit of the dust model for the comparison with the IR observations.

We use a simple mixture of amorphous silicate and amorphous $\mathrm{Al}_{2} \mathrm{O}_{3}$ (20\% by mass) dust grains. We use the optical constants of warm silicate grains (Suh 1999) for the models of $\tau_{10} \leq 3$ and cold silicate grains (Suh 1999) for $\tau_{10}>3$. We use the optical constants of amorphous $\mathrm{Al}_{2} \mathrm{O}_{3}$ (porous) obtained by Begemann et al. (1997) for all models. We assume that the bulk densities $\left(\rho_{d}\right)$ of the silicate grains and $\mathrm{Al}_{2} \mathrm{O}_{3}$ are 3.3 and $3.2 \mathrm{~g} \mathrm{~cm}^{-3}$, respectively. The radii of spherical dust grains are assumed to be $0.1 \mu \mathrm{m}$ uniformly.

Table 3 shows the list of theoretical mass-loss rates and color indices for various dust optical depths estimated from the radiative transfer models with a simple mixture of silicate and $\mathrm{Al}_{2} \mathrm{O}_{3}$ (20\% by mass) dust grains. We assume that the dust-to-gas ratio $(\delta)$ is 0.01

Table 3.

Theoretical mass-loss rates and color indices for various dust optical depths (a simple mixture of silicate and $\left.\mathrm{Al}_{2} \mathrm{O}_{3} ; \delta=0.01 ; V_{\text {exp }}=10 \mathrm{~km} / \mathrm{sec}\right)$.

\begin{tabular}{llll}
\hline$\tau_{10}$ & $K-[12]$ & {$[12]-[25]$} & $\dot{M}\left(M_{\odot} \mathrm{yr}^{-1}\right)$ \\
\hline 0.001 & 1.142 & 0.132 & $2.33 \mathrm{E}-09$ \\
0.01 & 1.381 & 0.282 & $2.34 \mathrm{E}-08$ \\
0.1 & 2.564 & 0.621 & $2.43 \mathrm{E}-07$ \\
0.5 & 3.962 & 0.814 & $1.36 \mathrm{E}-06$ \\
1 & 4.573 & 0.959 & $2.91 \mathrm{E}-06$ \\
3 & 5.742 & 1.419 & $9.89 \mathrm{E}-06$ \\
\hline 5 & 6.153 & 1.794 & $1.32 \mathrm{E}-05$ \\
10 & 8.016 & 2.266 & $2.99 \mathrm{E}-05$ \\
20 & 11.08 & 2.841 & $6.95 \mathrm{E}-05$ \\
30 & 13.18 & 3.233 & $1.16 \mathrm{E}-04$ \\
40 & 14.72 & 3.548 & $1.69 \mathrm{E}-04$ \\
60 & 17.07 & 4.056 & $2.91 \mathrm{E}-04$ \\
\hline
\end{tabular}




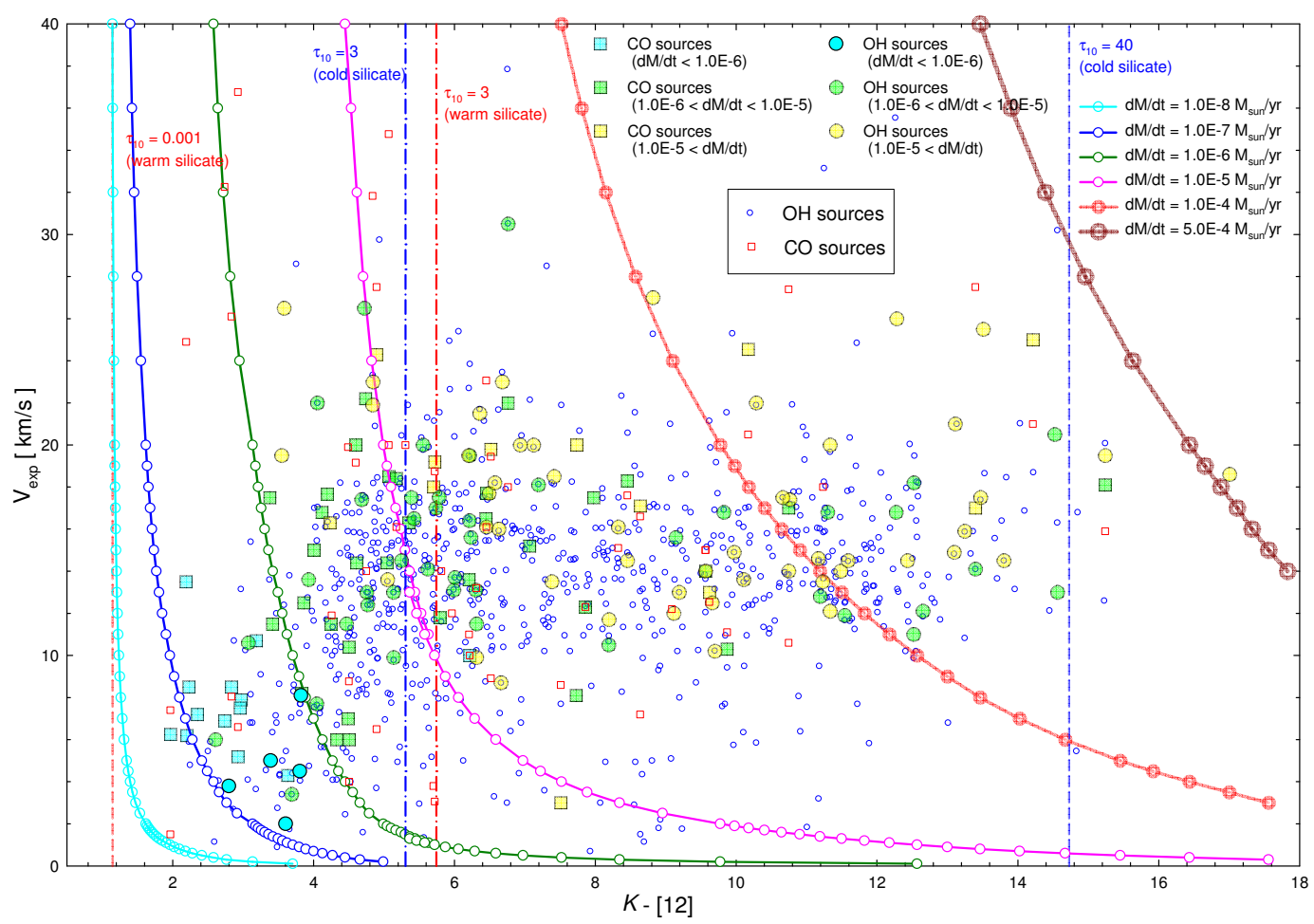

Fig. 2.- The $V_{\text {exp }}$ versus $K$-[12] diagram.

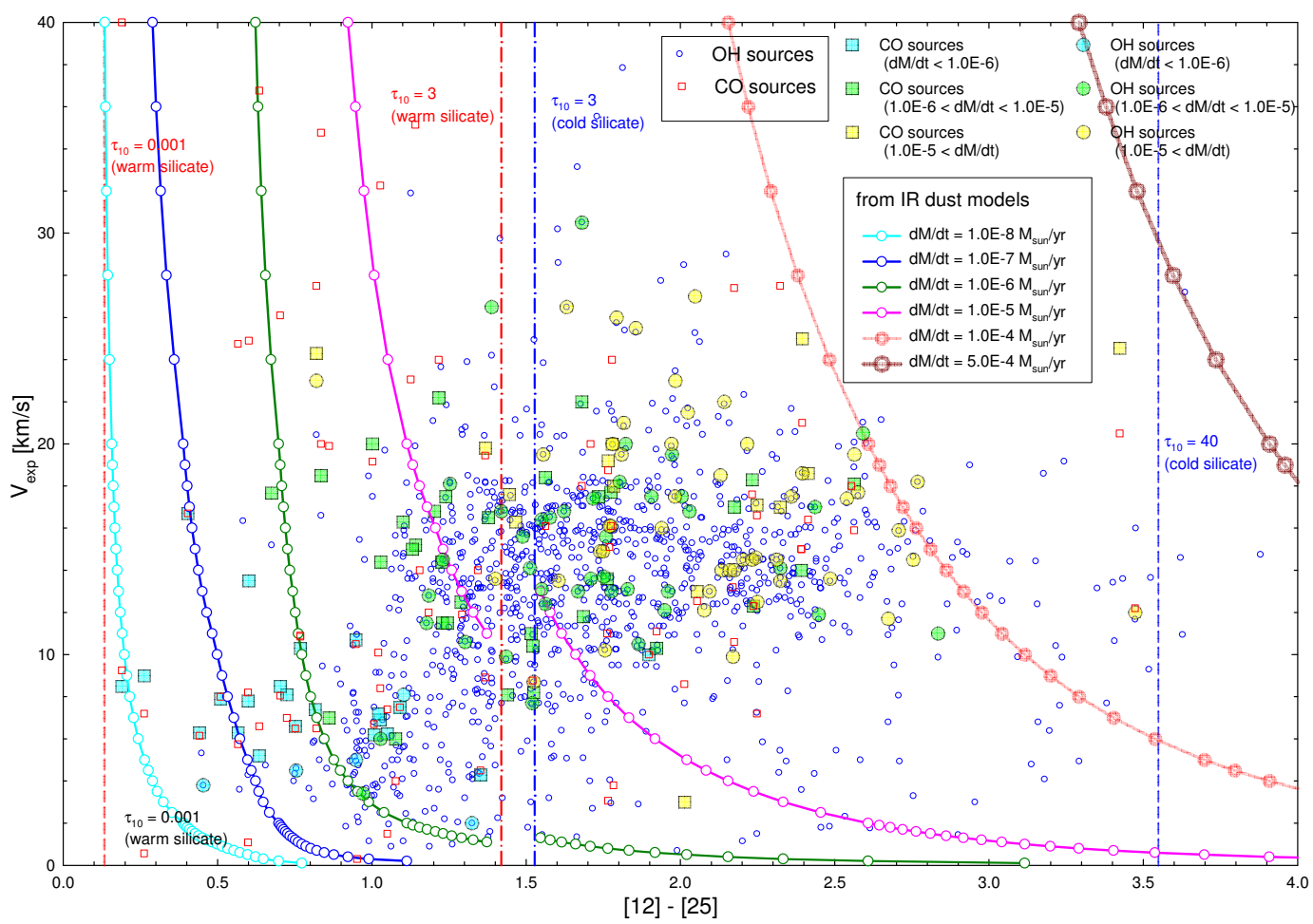

Fig. 3.- The $V_{\text {exp }}$ versus [12]-[25] diagram. 
and $V_{\text {exp }}=10 \mathrm{~km} / \mathrm{sec}$.

\section{COMPARISON OF THE THEORETICAL MODELS WITH THE OBSERVATIONS}

The stellar wind of an OH/IR star is driven by radiation pressure on dust grains and subsequent momentum transfer to the gas molecules via collisions (e.g., Kwok 1975). The mass-loss rate ( $\dot{M}$; see Eq. 2 which can be derived from a dust shell model, is related the dust shell optical depth $\left(\kappa_{\lambda} ; R_{i n} ; \rho_{d}\right)$ and $V_{\text {exp }}$. This can be compared with the ones obtained from radio observations.

\section{1 $V_{\text {exp }}$ - Color Diagrams}

In Figs. 2 and 3, we plot $V_{\exp }$ obtained from the $\mathrm{CO}$ and $\mathrm{OH}$ maser observations versus the IR color. We also plot the equi- $\dot{M}$ curves from the theoretical dust shell radiative transfer models. Each equi- $\dot{M}$ line is obtained by connecting the points of the same massloss rate by calculating the points on the diagrams by interpolating $\dot{M}$ and IR colors for given $V_{\text {exp }}$ from the dust shell models.

In Figs. 2 and 3, there is a general trend that $V_{\text {exp }}$ obtained from radio observations increases with the IR color for bluer objects (with smaller dust optical depths), while $V_{\text {exp }}$ levels off to a certain value for the redder objects. This behavior of the gas expansion velocity (i.e., $V_{\text {exp }}$ obtained from radio observations) can be understood on the basis of the efficiency of photon momentum transfer to the gas shell. When the massloss rate is small, any increase of the mass-loss rate causes a larger dust-gas coupling and so the momentum transfer increases. But when a critical value of the mass-loss rate is reached, further increase in the momentum transfer for accelerating gas is not possible (e.g., Kwok 1975; Stefen et al. 1997). Large scatters in $V_{\text {exp }}$ could be due to pulsation (e.g., Suh 2004).

There are overlaps and gaps in the equi- $\dot{M}$ curves in $V_{\text {exp }}$ versus color diagrams at the color indices which correspond to $\tau_{10}=3$. This is because we use different input parameters (the dust opacity; the temperature of the central star) for two different conditions $\left(\tau_{10} \leq 3\right.$ and $\tau_{10}>3$; see Section 3.1).

In Figs. 2 and 3, the observed mass-loss rates start to rise close from the theoretical line of $10^{-8} M_{\odot} / y r$ to the line of $10^{-4} M_{\odot} / y r$ determined from the dust models. Stefen et al. (1997) estimated an upper limit of $(3-10) \times 10^{-4} M_{\odot} / y r$ for dust driven winds depending on the various stellar and dust parameters.

Generally, theoretical mass-loss rate curves can explain the observed mass-loss rates and expansion velocities fairly well. As noted by Suh \& Kwon (2011), the dust opacity (silicate and $\mathrm{Al}_{2} \mathrm{O}_{3}$ ) used for this paper can reproduce both $K$-[12] and [12]-[25] better than the dust opacity using only silicate.

\section{2 $\dot{M}$ - Color Diagrams}

In Figs. 4 and 5, we plot the diagrams of the massloss rates obtained from $\mathrm{OH}$ and $\mathrm{CO}$ observations versus the IR color $(K-[12]$ and [12]-[25]) for OH/IR stars. We also plot the equi- $V_{\text {exp }}$ curves derived from the theoretical dust radiative transfer models.

The observed mass-loss rate increases with the IR color until it reaches about $10^{-5} M_{\odot} / y r$. After that, the mass-loss rate tends to maintain a certain level.

In the region of low mass-loss rates $\left(\tau_{10} \leq 3\right)$, the theoretical mass-loss rates derived from the dust shell models generally agree with the radio observations.

In the region of high mass-loss rate $\left(\tau_{10}>3\right)$, the theoretical mass-loss rates are obviously larger than the ones obtained from radio observations especially for higher dust optical depths. As we commented in Section 4.1, this could be because the photon momentum transfer to the gas shell is not possible for the physical condition of high mass-loss rates.

For high mass-loss rate $\mathrm{OH} / \mathrm{IR}$ stars, the outer gas shell radius which is measured by radio observations is smaller than the outer dust shell radius which is measured by IR observations. For OH 26.5+0.6, Suh \& Kwon (2013) could reproduce the observed image at $8.7 \mu \mathrm{m}$ obtained by Chesneau et al. (2005) using the dust model image of $R_{\text {out }}=3 \times 10^{4}$ AU. However, the $\mathrm{CO}(\mathrm{J}=1-0)$ image size of the object is much smaller (4700 AU; Fong et al. 2002). This may mean that the gas mass-loss is not as effective as the dust mass-loss for high mass-loss rate $\mathrm{OH} / \mathrm{IR}$ stars. Alternative explanations could be the effects of different dust-to-gas ratios and/or a superwind (see Section 1).

The dust-to-gas ratio could be larger than 0.01 for the high mass-loss rate $\mathrm{OH} / \mathrm{IR}$ stars which are massive, younger, and metal-rich. In Figs. 4 and 5 , the model results with a larger dust-to-gas ratio $(\delta=0.02)$ are also shown for high mass-loss rates $\left(\tau_{10} \geq 15\right)$. A superwind would effectively make abrupt changes of the mass-loss rate depending on the location (e.g., Suh \& Jones 1997).

\section{3 $\dot{M}$ - Period Diagram}

On Fig. 6, the mass-loss rates obtained from radio observations are compared with the pulsation periods for $26 \mathrm{OH}$ sources (Chen et al. 2001; Zhang et al. 2010) and 15 CO sources (De Beck et al. 2010).

We find that a linear relation fits the $\mathrm{OH} / \mathrm{IR}$ stars in our sample with periods shorter than about 800 days. However, $\dot{M}$ obtained from radio observation levels off to an almost constant value of $4 \times 10^{-5} M_{\odot} / y r$ for longer periods. This could be due to relatively ineffective gas mass-loss for high mass-loss rate $\mathrm{OH} / \mathrm{IR}$ stars (see Section 4.2). Even though it is difficult to determine the meaningful saturation mass-loss rate and cut-off period due to the lack of data points, a similar relationship was found for different samples of LPVs (e.g., De Beck et al. 2010). 


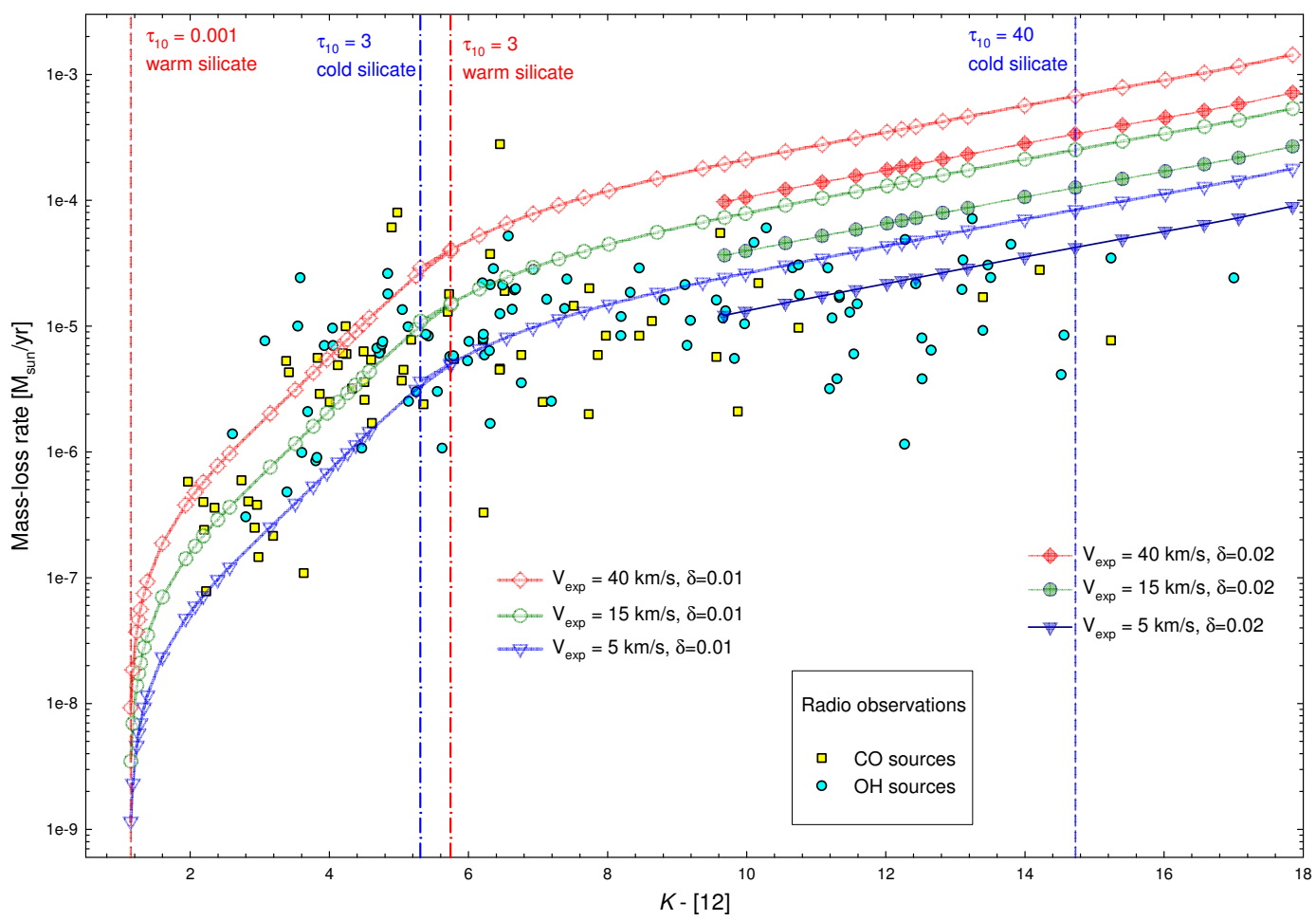

Fig. 4. - The mass-loss rate versus $K$-[12] diagram.

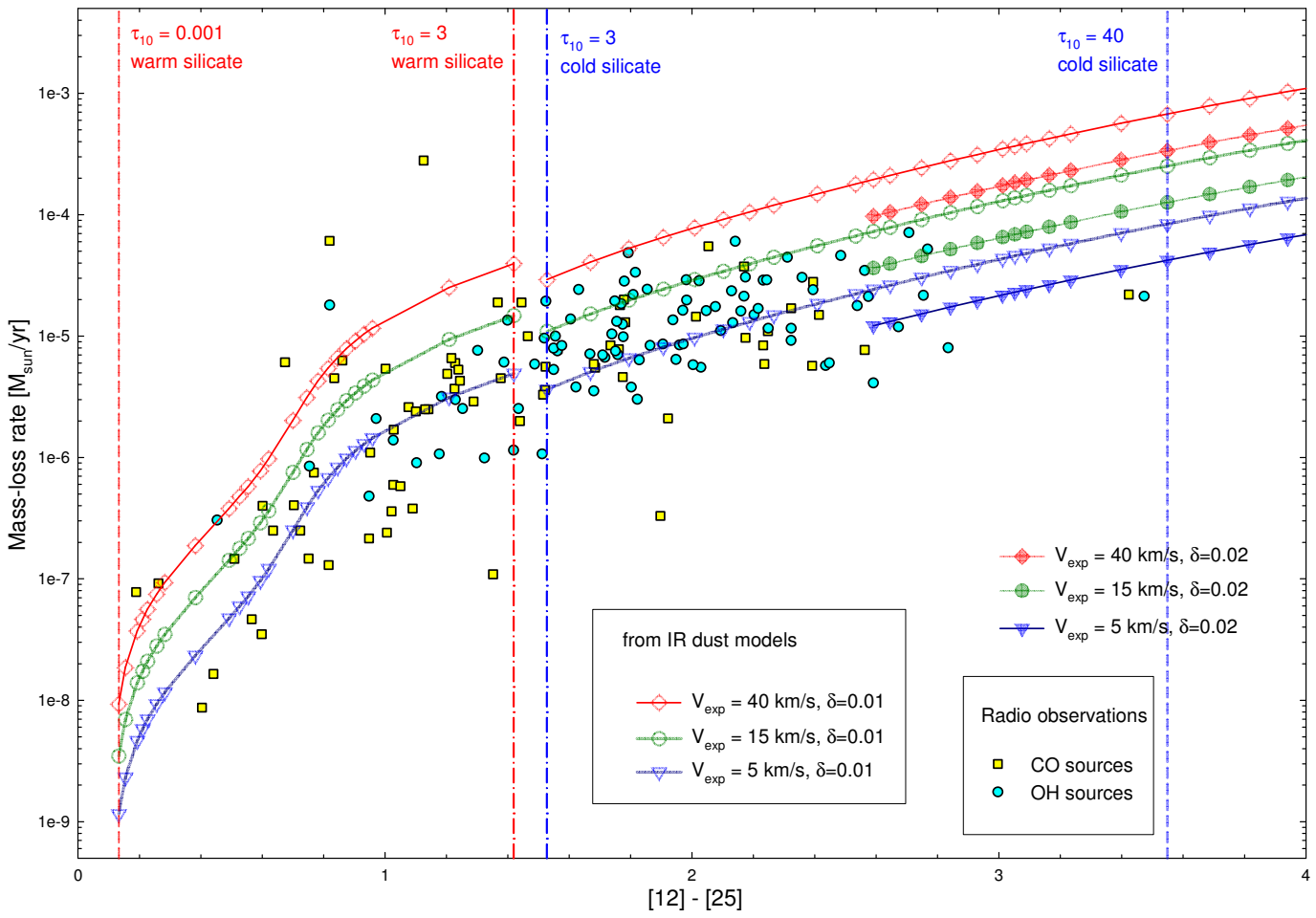

Fig. 5.- The mass-loss rate versus [12]-[25] diagram. 


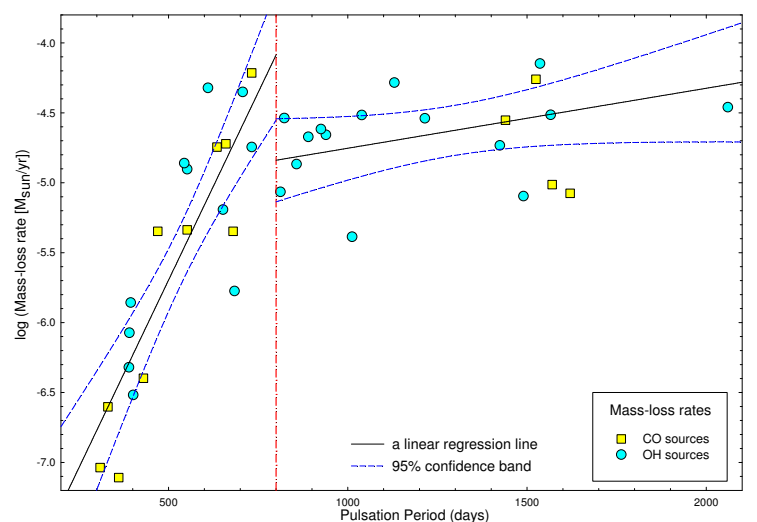

Fig. 6.- Comparison of the mass-loss rates obtained from radio observations with the pulsation periods for $\mathrm{OH} / \mathrm{IR}$ stars.

\section{SUMMARY}

We have collected radio observational data for a sample of $1533 \mathrm{OH} / \mathrm{IR}$ stars listed in Suh \& Kwon (2011). We have compiled data of the expansion velocity and mass-loss rate for $1259 \mathrm{OH}$ maser, $76 \mathrm{CO}(\mathrm{J}=1-$ $0)$, and $55 \mathrm{CO}(\mathrm{J}=2-1)$ emission sources.

We have used a dust radiative transfer model for the dust shell to calculate the mass-loss rate curves as well as the IR color indices ( $K-[12]$ and [12]-[25]). The theoretical mass-loss rates are compared with those obtained by radio observations. We have found that a simple mixture of amorphous silicate and amorphous $\mathrm{Al}_{2} \mathrm{O}_{3}$ (20\% by mass) dust grains can explain the observations fairly well.

On $V_{\text {exp }}$ - color diagrams, the mass-loss rates and expansion velocities from radio observations are reasonably in the range of the theoretical dust shell models corresponding to $\dot{M}=10^{-8} M_{\odot} / y r-10^{-4} M_{\odot} / y r$. The results indicate that the dust radiative transfer models derived from IR observations generally agree with the radio observations.

On $\dot{M}$ - color diagrams, the mass-loss rates obtained from radio observations are underestimated for high mass-loss rate $\mathrm{OH} / \mathrm{IR}$ stars, compared to the mass-loss rates derived from the dust models. This could be because the photon momentum transfer to the gas shell is not possible for the high mass-loss rates. Alternative explanations could be the effects of different dust-togas ratios and/or a superwind. Further investigations on a detailed hydrodynamical model of gas and dust considering the effect of a superwind and more dust species would be necessary to fully understand the disagreements.

\section{ACKNOWLEDGMENTS}

This work was supported by the research grant of Chungbuk National University in 2012.

\section{REFERENCES}

Begemann, B., Dorschner, J., Henning, T., Mutschke, H., Guertler, J., Koempe, C., \& Nass, R. 1997, Aluminum Oxide and the Opacity of Oxygen-Rich Circumstellar Dust in the 12-17 Micron Range, ApJ, 476, 199

Chen, P. S., Szczerba, R., Kwok, S., \& Volk, K. 2001, Properties of OH/IR Stars with IRAS LRS Spectra, A\&A, 368, 1006

Chengalur, J. N., Lewis, B. M., Eder, J., \& Terzian, Y. 1993, New OH/IR Stars from Color-Selected IRAS Sources. 3: A Complete Survey, ApJSS, 89, 189

Chesneau, O., Verhoelst, T., Lopez, B., et al. 2005, The Mid-IR Spatially Resolved Environment of $\mathrm{OH}$ 26.5+0.6 at Maximum Luminosity, A\&A, 435, 563

David, P., Le Squeren, A. M., \& Sivagnanam, P. 1993, An OH Satellite Line Maser Survey of Cool IRAS Sources and Circumstellar Envelope Evolution, A\&A, 277, 453

De Beck, E., Decin, L., de Koter, A., Justtanont, K., Verhoelst, T., Kemper, F., \& Menten, K. M. 2010, Probing the Mass-Loss History of AGB and Red Supergiant Stars from CO Rotational Line Profiles. II. CO Line Survey of Evolved Stars: Derivation of Mass-Loss Rate Formulae, A\&A, 523, 18

Draine, B. T., Dale, D. A., Bendo, G., et al. 2007, Dust Masses, PAH Abundances, and Starlight Intensities in the SINGS Galaxy Sample, ApJ, 663, 866

Eder, J., Lewis, B. M., \& Terzian, Y. 1988, New OH/IR Stars from Color-Selected IRAS Sources - $1612 \mathrm{MHz}$ Observations, ApJS, 66, 183

Fong, D., Justtanont, K., Meixner, M., \& Campbell, M. T. 2002, Imaging the Circumstellar Envelope of OH 26.5+0.6, A\&A, 396, 851

Groenewegen, M. A. T., \& de Jong, T. 1998, CO Observations and Mass Loss of MS- and S-Stars, A\&A, 337,797

Groenewegen, M. A. T., Baas, F., Blommaert, J. A. D. L., Stehle, R., Josselin, E., \& Tilanus, R. P. J. 1999, Millimeter and Some Near Infra-Red Observations of Short-Period Miras and Other AGB Stars, A\&AS, 140, 197

Iben, I. Jr. 1981, The Carbon Star Mystery - Why Do the Low Mass Ones Become Such, and Where Have All the High Mass Ones Gone, ApJ, 246, 278

Ivezić, A., \& Elitzur, M. 1997, Self-Similarity and Scaling Behaviour of Infrared Emission from Radiatively Heated Dust - I. Theory, MNRAS, 287, 799

Knapp, G. R., Young, K., Lee, E., \& Jorissen, A. 1998, Multiple Molecular Winds in Evolved Stars. I. A Survey of CO (2-1) and CO (3-2) Emission from 45 Nearby AGB Stars, ApJS, 117, 209 
Kemper, F., Stark, R., Justtanont, K., de Koter, A., Tielens, A. G. G. M., Waters, L. B. F. M., Cami, J., \& Dijkstra, C. 2003, Mass Loss and Rotational CO Emission from AGB Stars, A\&A, 407, 609

Kwok, S. 1975, Radiation Pressure on Grains as a Mechanism for Mass Loss in Red Giants, ApJ, 198, 583

Kwon, Y.-J., \& Suh, K.-W. 2012, Properties of OH, $\mathrm{SiO}$, and $\mathrm{H}_{2} \mathrm{O}$ Maser Emission in O-Rich AGB Stars, JKAS, 45, 139

Le Squeren, A. M., Sivagnanam, P., Dennefeld, M., \& David, P. 1992, A Complete Survey of OH/IR Objects from the IRAS LRS Sources within a Domain of the Color Diagram, A\&A, 254, 133

Lewis, B. M. 1994, Supplement to the Arecibo 1612 $\mathrm{MHz}$ Survey of Color-Selected IRAS Sources, ApJS, 93,549

Lewis, B. M., Eder, J., \& Terzian, Y. 1990, New OH/IR Stars from Color-Selected IRAS Sources. II - An Unbiased 1612 MHz Survey, ApJ, 362, 634

Loup, C., Forveille, T., Omont, A., \& Paul, J. F. 1993, CO and HCN Observations of Circumstellar Envelopes. A Catalogue - Mass Loss Rates and Distributions, A\&AS, 99, 291

Nyman, L.-A., Booth, R. S., Carlstrom, U., Habing, H. J., Heske, A., Sahai, R., Stark, R., van der Veen, W. E. J. C., \& Winnberg, A. 1992, A Survey of Circumstellar CO Emission from a Sample of IRAS Point Sources, A\&AS, 93, 121

Olofsson, H., Eriksson, K., Gustafsson, B., \& Carlstrom, U. 1993, A Study of Circumstellar Envelopes around Bright Carbon Stars. I - Structure, Kinematics, and Mass-Loss Rate, ApJS, 87, 267

Renzini, A. 1981, in Physical Processes in Red Giants, ed. I. Iben \& A. Renzini (Dordrecht: Reidel), 431

Sevenster, M. N., Chapman, J. M., Habing, H. J., Killeen, N. E. B., \& Lindqvist, M. 1997a, The ATCA/VLA OH 1612 MHz Survey. I. Observations of the Galactic Bulge Region, A\&AS, 122, 79

Sevenster, M. N., Chapman, J. M., Habing, H. J., Killeen, N. E. B., \& Lindqvist, M. 1997b, The ATCA/VLA OH 1612 MHz Survey. II. Observations of the Galactic Disk Region, A\&AS, 124, 509

Sevenster, M. N., van Langevelde, H. J., Moody, R. A., Chapman, J. M., Habing, H. J., \& Killeen, N. E. B. 2001, The ATCA/VLA OH 1612 MHz Survey. III. Observations of the Northern Galactic Plane, A\&A, 366,481

Sivagnanam, P., Le Squeren, A. M., Minh, F. T., \& Braz, M. A. 1990, An OH Survey of Mira-Like Objects from the IRAS Point Source Catalog, A\&A, 233, 112

Stefen, M., Szczerba, R., Men'schikov, A., et al. 1997, Hydrodynamical Models and Synthetic Spectra of Circumstellar Dust Shells around AGB Stars, A\&AS, 126, 39
Suh, K.-W. 1999, Optical Properties of the Silicate Dust Grains in the Envelopes around Asymptotic Giant Branch Stars, MNRAS, 304, 389

Suh, K.-W. 2004, Pulsation Phase-Dependent Dust Shell Models for Oxygen-Rich Asymptotic Giant Branch Stars, ApJ, 615, 485

Suh, K.-W., \& Jones, T. J. 1997, Superwind Models for the Dust Shells around OH/IR Stars, ApJ, 479, 918

Suh, K.-W., \& Kwon, Y.-J. 2011, Infrared Two-Colour Diagrams for AGB Stars Using AKARI, MSX, IRAS and Near-Infrared Data, MNRAS, 417, 3047

Suh, K.-W., \& Kwon, Y.-J. 2013, Water Ice in High Mass-Loss Rate OH/IR Stars, ApJ, 762, 113.

te Lintel Hekkert, P., Caswell, J. L., Habing, H. J., Haynes, R. F., Haynes, R. F., \& Norris, R. P. 1991, $1612 \mathrm{MHz}$ OH Survey of IRAS Point Sources. I Observations Made at Dwingeloo, Effelsberg and Parkes, A\&AS, 90, 327

Vassiliadis, E., \& Wood, P. R. 1993, Evolution of Low- and Intermediate-Mass Stars to the End of the Asymptotic Giant Branch with Mass Loss, ApJ, 413, 641

Winters, J. M., Le Bertre, T., Jeong, K. S., Nyman, L.-A., \& Epchtein, N. 2003, Mass-Loss from Dusty, Low Outflow-Velocity AGB Stars. I. Wind Structure and Mass-Loss Rates, A\&A, 409, 715

Wood, P. R. 1990, in From Miras to Planetary Nebulae : Which Path Evolution, ed. M. O. Minnessier \& A. Omont (Gif sur Yvette: Editions Frontières), 67

Zhang, H.-J., Zhou, J.-J., Dong, G.-L., Esimbek, J., \& Mu, J.-M. 2010, The Properties of a Large Sample of $\mathrm{OH} / \mathrm{IR}$ Stars, S Stars and C-Rich AGB Stars, ApSS, 330,23 